Proceedings of The Ninth International

Workshop on Diff. Geom. 9(2005) 31-39

\title{
On generalized Sasakian-space-forms
}

\section{Alfonso Carriazo}

From a joint work with David E. Blair and PABlo Alegre

Department of Geometry and Topology, Faculty of Mathematics, University of Sevilla, Apdo. Correos 1160, 41080 Sevilla, Spain

e-mail : carriazo@us.es

(2000 Mathematics Subject Classification : 53C25, 53D15.)

Abstract. We study contact metric and trans-Sasakian generalized Sasakian-space-forms. We also give some interesting examples of generalized Sasakian-space-forms by using warped products and conformal changes of metric.

\section{Introduction}

The study of the curvature tensor $R$ of a Riemannian manifold and the elements which allow us to determine it has always been a main topic in differential geometry. In this sense, Sasakian-space-forms play an important role in contact Riemannian geometry.

A Sasakian manifold $(M, \phi, \xi, \eta, g)$ is said to be a Sasakian-space-form if all the $\phi$-sectional curvatures $K(X \wedge \phi X)$ are equal to a constant $c$, where $K(X \wedge \phi X)$ denotes the sectional curvature of the section spanned by the unit vector field $X$, orthogonal to $\xi$, and $\phi X$. In such a case, the Riemann curvature tensor of $M$ is given by

$$
\begin{aligned}
R(X, Y) Z= & \frac{c+3}{4}\{g(Y, Z) X-g(X, Z) Y\} \\
+ & \frac{c-1}{4}\{g(X, \phi Z) \phi Y-g(Y, \phi Z) \phi X+2 g(X, \phi Y) \phi Z\} \\
+ & \frac{c-1}{4}\{\eta(X) \eta(Z) Y-\eta(Y) \eta(Z) X \\
& +g(X, Z) \eta(Y) \xi-g(Y, Z) \eta(X) \xi\}
\end{aligned}
$$

These spaces can be modeled, depending on $c>-3, c=-3$ or $c<-3$.

As a natural generalization of these manifolds, P. Alegre, D. E. Blair and the author introduced in [1] the notion of generalized Sasakian-space-forms. They were defined as almost contact metric manifolds with Riemann curvature tensors satisfying an equation similar to (1.1), in which the constant quantities, $(c+3) / 4$ and 
$(c-1) / 4$ are replaced by differentiable functions, i.e., such that

$$
\begin{aligned}
R(X, Y) Z= & f_{1}\{g(Y, Z) X-g(X, Z) Y\} \\
+ & f_{2}\{g(X, \phi Z) \phi Y-g(Y, \phi Z) \phi X+2 g(X, \phi Y) \phi Z\} \\
+ & f_{3}\{\eta(X) \eta(Z) Y-\eta(Y) \eta(Z) X \\
& +g(X, Z) \eta(Y) \xi-g(Y, Z) \eta(X) \xi\} .
\end{aligned}
$$

Let us notice that a generalized Sasakian-space-form is not, in general, a Sasakian manifold, just like a generalized complex-space-form is not always a Kaehler manifold (see [8, 9]).

In this lecture, we will first study the structure of a generalized Sasakian-spaceform. In particular, we will focus on both contact metric and trans-Sasakian ones. On the other hand, we will show some procedures to obtain interesting examples (i.e., examples with non-constant functions $f_{1}, f_{2}, f_{3}$ ) by using warped products and conformal changes of metric. For more details and the proofs of the theorems presented in the following sections, we refer to $[1,2]$.

Acknowledgements The author is partially supported by the MEC-FEDER grant MTM2004-04934-C04-04, the PAI group FQM-327 and the BK21 Proj. of KRF. He wants to express his deepest gratitude to Prof. Young Jin Suh and Prof. Young Ho Kim for the invitation to participate in this Workshop and the warm hospitality received during his visit to Kyungpook National University.

\section{Preliminaries}

In this section, we recall some definitions and basic formulas which we will use later. For more background on almost contact metric manifolds, we recommend the reference [3].

An odd-dimensional Riemannian manifold $(M, g)$ is said to be an almost contact metric manifold if there exist on $M$ a $(1,1)$ tensor field $\phi$, a vector field $\xi$ (called the structure vector field) and a 1-form $\eta$ such that $\eta(\xi)=1, \phi^{2}(X)=-X+\eta(X) \xi$ and $g(\phi X, \phi Y)=g(X, Y)-\eta(X) \eta(Y)$, for any vector fields $X, Y$ on $M$. In particular, in an almost contact metric manifold we also have $\phi \xi=0$ and $\eta \circ \phi=0$.

Such a manifold is said to be a contact metric manifold if $\mathrm{d} \eta=\Phi$, were $\Phi(X, Y)=g(X, \phi Y)$ is called the fundamental 2-form of $M$. If, in addition, $\xi$ is a Killing vector field, then $M$ is said to be a $K$-contact manifold. It is well-known that a contact metric manifold is a $K$-contact manifold if and only if $\nabla_{X} \xi=-\phi X$, for any vector field $X$ on $M$. On the other hand, the almost contact metric structure of $M$ is said to be normal if $[\phi, \phi](X, Y)=-2 d \eta(X, Y) \xi$, for any $X, Y$, where $[\phi, \phi]$ denotes the Nijenhuis torsion of $\phi$, given by $[\phi, \phi](X, Y)=$ $\phi^{2}[X, Y]+[\phi X, \phi Y]-\phi[\phi X, Y]-\phi[X, \phi Y]$. A normal contact metric manifold is called a Sasakian manifold. It can be proved that an almost contact metric manifold 
is Sasakian if and only if

$$
\left(\nabla_{X} \phi\right) Y=g(X, Y) \xi-\eta(Y) X,
$$

for any $X, Y$.

In [7], J. A. Oubiña introduced the notion of a trans-Sasakian manifold. An almost contact metric manifold $M$ is a trans-Sasakian manifold if there exist two functions $\alpha$ and $\beta$ on $M$ such that

$$
\left(\nabla_{X} \phi\right) Y=\alpha(g(X, Y) \xi-\eta(Y) X)+\beta(g(\phi X, Y) \xi-\eta(Y) \phi X),
$$

for any $X, Y$ on $M$. If $\beta=0, M$ is said to be an $\alpha$-Sasakian manifold. Sasakian manifolds appear as examples of $\alpha$-Sasakian manifolds, with $\alpha=1$. If $\alpha=0, M$ is said to be a $\beta$-Kenmotsu manifold. Kenmotsu manifolds are particular examples with $\beta=1$. If both $\alpha$ and $\beta$ vanish, then $M$ is a cosymplectic manifold. Actually, in [6], Marrero showed that a trans-Sasakian manifold of dimension greater than or equal to 5 is either $\alpha$-Sasakian, $\beta$-Kenmotsu or cosymplectic.

In the following two sections we will show some results giving us more information about the relationship between the structure on a generalized Sasakian-spaceform and the functions $f_{1}, f_{2}, f_{3}$. In this sense, we have the following theorem from [5], which we adapt to our notation:

Theorem 2.1. ([5]) Let $(M, \phi, \xi, \eta, g)$ be a connected generalized Sasakian-spaceform with $f_{2}=f_{3}$ not identically zero. If $\operatorname{dim}(M) \geq 5$ and $g\left(X, \nabla_{X} \xi\right)=0$ for any vector field $X$ orthogonal to $\xi$, then $f_{1}$ and $f_{2}$ are constant functions and $f_{1}-f_{2} \geq 0$. Moreover, if $f_{1}-f_{2}=0$, then $(M, \phi, \xi, \eta, g)$ is a cosymplectic-space-form and if $f_{1}-f_{2}=\alpha^{2}>0$ then $(M, \phi, \xi, \eta, g)$ or $(M,-\phi, \xi, \eta, g)$ is an $\alpha$-Sasakian manifold with constant $\phi$-sectional curvature $c$ and a generalized Sasakian-space-form with $f_{1}=\left(c+3 \alpha^{2}\right) / 4$ and $f_{2}=f_{3}=\left(c-\alpha^{2}\right) / 4$.

\section{Contact metric generalized Sasakian-space-forms}

By using (1.2) and the well-known fact which establishes that in a $K$-contact manifold, the sectional curvature of any plane section containing $\xi$ is equal to 1 , we can first prove:

Proposition 3.1. ([1]) Let $M\left(f_{1}, f_{2}, f_{3}\right)$ be a generalized Sasakian-space-form. If $M$ is a K-contact manifold, then $f_{3}=f_{1}-1$.

Moreover, it is well-known that any Sasakian manifold is a $K$-contact manifold. For a generalized Sasakian-space-form, the converse is also true: 
Theorem 3.1. ([1]) Every generalized Sasakian-space-form with a K-contact structure is a Sasakian manifold.

For a contact metric generalized Sasakian-space-form, we can obtain the following theorems:

Theorem 3.2. ([1]) Let $M\left(f_{1}, f_{2}, f_{3}\right)$ be a generalized Sasakian-space-form. If $M$ is a contact metric manifold with $f_{3}=f_{1}-1$, then it is a Sasakian manifold.

Theorem 3.3. ([1]) Let $M\left(f_{1}, f_{2}, f_{3}\right)$ be a generalized Sasakian-space-form. If $M$ is a contact metric manifold, then $f_{1}-f_{3}$ is constant on $M$.

Let us notice that the assumption $f_{3}=f_{1}-1$ in Theorem 3.2 is coherent with Theorem 3.3. In that case, the constant is just 1 .

On the other hand, by working with some curvature identities, we can also prove:

Theorem 3.4. ([1]) Let $M\left(f_{1}, f_{2}, f_{3}\right)$ be a generalized Sasakian-space-form. If $M$ is a Sasakian manifold, then $f_{2}=f_{3}=f_{1}-1$.

Thus, by using the obstruction established in Theorem 2.1, we deduce that, in dimensions greater than or equal to 5 , any contact metric connected generalized Sasakian-space-form, such that $f_{3}=f_{1}-1$, must be a Sasakian-space-form. In particular, this fact is true for either $K$-contact or Sasakian generalized Sasakianspace-forms.

But, is it still possible to find interesting examples of contact metric generalized Sasakian-space-forms non-satisfying the above conditions? To answer this question, we have to point out that a contact metric generalized Sasakian-space-form is a particular case of $(\kappa, \mu)$-space, with $\kappa=f_{1}-f_{3}$ and $\mu=0$. Let us recall that a contact metric manifold is said to be a $(\kappa, \mu)$-space ([4]) if

$$
R(X, Y) \xi=\kappa\{\eta(Y) X-\eta(X) Y\}+\mu\{\eta(Y) h X-\eta(X) h Y\},
$$

for any vector fields $X, Y$ on $M$, where $h X=1 / 2\left(L_{\xi} \phi\right) X, L$ being the usual Lie derivative.

Then, by virtue of Theorem 1 of [4] we can obtain the following results:

Lemma 3.1. ([2]) If $M\left(f_{1}, f_{2}, f_{3}\right)$ is a non-Sasakian contact metric generalized Sasakian-space-form, with dimension greater than or equal to 5 , then $f_{2}=\kappa$.

Lemma 3.2. ([2]) If $M\left(f_{1}, f_{2}, f_{3}\right)$ is a non-Sasakian contact metric generalized Sasakian-space-form, with dimension greater than or equal to 5 , then $f_{2}=0$. 
Lemma 3.3. ([2]) If $M\left(f_{1}, f_{2}, f_{3}\right)$ is a non-Sasakian contact metric generalized Sasakian-space-form, with dimension greater than or equal to 5 , then $f_{1}=f_{3}=0$.

It follows from 1.2 and the above lemmas that a non-Sasakian contact metric generalized Sasakian-space-form, with dimension greater than or equal to 5 should be a flat manifold. But, as a contact metric manifold with such dimension cannot be flat, we have:

Theorem 3.5. ([2]) Any contact metric generalized Sasakian-space-form $M\left(f_{1}, f_{2}, f_{3}\right)$, with dimension greater than or equal to 5 , is a Sasakian manifold. Therefore, $f_{1}$, $f_{2}$ and $f_{3}$ must be constant functions.

And, what can we say about 3-dimensional contact metric generalized Sasakianspace-forms? First, let us mention that the writing of the curvature tensor of a 3 -dimensional generalized Sasakian-space-form is not unique. Actually, if $M^{3}$ is an almost contact metric manifold such that its curvature tensor can be written as

$$
R(X, Y) Z=f_{1} R_{1}(X, Y) Z+f_{2} R_{2}(X, Y) Z+f_{3} R_{3}(X, Y) Z
$$

and

$$
R(X, Y) Z=f_{1}^{*} R_{1}(X, Y) Z+f_{2}^{*} R_{2}(X, Y) Z+f_{3}^{*} R_{3}(X, Y) Z,
$$

where $R_{i}(X, Y) Z$ denotes the corresponding term in (1.2), then the functions $f_{i}$ and $f_{i}^{*}$ are related as follows,

$$
f_{1}^{*}=f_{1}+f, \quad f_{2}^{*}=f_{2}-f / 3, \quad f_{3}^{*}=f_{3}+f
$$

where $f$ is a function on $M$. Conversely, if $M^{3}\left(f_{1}, f_{2}, f_{3}\right)$ is a generalized Sasakianspace-form and we define the functions $f_{i}^{*}$ as above, for any function $f$ on $M$, then it is also a generalized Sasakian-space-form $M^{3}\left(f_{1}^{*}, f_{2}^{*}, f_{3}^{*}\right)$. Therefore, in order to consider an unique writing of the curvature tensor of a three-dimensional generalized Sasakian-space-form, we will chose that satisfying $f_{2}=0$.

Now, we can show the following results from [2]:

Proposition 3.2. ([2]) If $M^{3}\left(f_{1}, f_{2}, f_{3}\right)$ is a 3-dimensional, non-Sasakian, contact metric generalized Sasakian-space-form, then $2 f_{1}+3 f_{2}-f_{3}=0$.

Theorem 3.6. ([2]) If $M^{3}\left(f_{1}, f_{2}, f_{3}\right)$ is a 3-dimensional, non-Sasakian, contact metric generalized Sasakian-space-form, then we can write

$$
R(X, Y) Z=-\kappa R_{1}(X, Y) Z-2 \kappa R_{3}(X, Y) Z,
$$

for any $X, Y, Z$ vector fields on $M$, where $\kappa<1$ is a constant. 


\section{Examples}

Even if from the results in the previous section we could think that it must be difficult to find examples of generalized Sasakian-space-forms with non-constant functions $f_{1}, f_{2}, f_{3}$, in this section we will show some construction procedures to obtain them.

Given an almost Hermitian manifold $(N, J, G)$, we consider the warped product $M=\mathbb{R} \times_{f} N$, where $f>0$ is a function on $\mathbb{R}$. This manifold can be endowed with an almost contact metric structure $\left(\phi, \xi, \eta, g_{f}\right)$. In fact,

$$
g_{f}=\pi^{*}\left(g_{\mathbb{R}}\right)+(f \circ \pi)^{2} \sigma^{*}(G),
$$

is the warped product metric, where $\pi$ and $\sigma$ are the projections from $\mathbb{R} \times N$ on $\mathbb{R}$ and $N$, respectively; $\phi(X)=\left(J \sigma_{*} X\right)^{*}$, for any vector field $X$ on $M$, and $\xi=\partial / \partial t$, where $t$ denotes the coordinate of $\mathbb{R}$. Then, we have:

Theorem 4.1. ([1]) Let $N\left(F_{1}, F_{2}\right)$ be a generalized complex-space-form. Then, the warped product $M=\mathbb{R} \times_{f} N$, endowed with the almost contact metric structure $\left(\phi, \xi, \eta, g_{f}\right)$, is a generalized Sasakian-space-form $M\left(f_{1}, f_{2}, f_{3}\right)$ with functions:

$$
f_{1}=\frac{\left(F_{1} \circ \pi\right)-f^{\prime 2}}{f^{2}}, \quad f_{2}=\frac{F_{2} \circ \pi}{f^{2}}, \quad f_{3}=\frac{\left(F_{1} \circ \pi\right)-f^{\prime 2}}{f^{2}}+\frac{f^{\prime \prime}}{f} .
$$

In particular, if $N(c)$ is a complex-space-form, we obtain the generalized Sasakian-space-form

$$
M\left(\frac{c-4 f^{\prime 2}}{4 f^{2}}, \frac{c}{4 f^{2}}, \frac{c-4 f^{\prime 2}}{4 f^{2}}+\frac{f^{\prime \prime}}{f}\right) .
$$

Hence, for example, the warped products $\mathbb{R} \times_{f} \mathbb{C}^{n}, \mathbb{R} \times_{f} \mathbb{C P}^{n}(4)$ and $\mathbb{R} \times_{f} \mathbb{C H} \mathbb{H}^{n}(-4)$ are generalized Sasakian-space-forms.

Moreover, we can obtain more examples by using conformal and related changes of metric. We just summarize now the most important results concerning these procedures. For more results and all the details, we refer once again to [1].

Given an almost contact metric manifold $(M, \phi, \xi, \eta, g)$, if we now consider a conformal change of metric

$$
g^{*}=\rho^{2} g,
$$

where $\rho$ is a positive function on $M$, then it is easy to prove that $\left(M, \phi^{*}, \xi^{*}, \eta^{*}, g^{*}\right)$ is also an almost contact metric manifold, where we put

$$
\phi^{*}=\phi, \quad \xi^{*}=\frac{1}{\rho} \xi, \quad \eta^{*}=\rho \eta .
$$


Theorem 4.2. ([1]) Given a complex-space-form $N(c)$ and two positive functions $f=f(t), \rho=\rho(t)$, the conformal change of metric with function $\rho$ endows the warped product $M=\mathbb{R} \times{ }_{f} N(c)$ with the structure of a generalized Sasakian-spaceform $M\left(f_{1}^{*}, f_{2}^{*}, f_{3}^{*}\right)$ with functions

$$
\begin{gathered}
f_{1}^{*}=\frac{1}{\rho^{2}}\left(\frac{c}{4 f^{2}}-\left(\frac{f^{\prime}}{f}+\frac{\rho^{\prime}}{\rho}\right)^{2}\right), \quad f_{2}^{*}=\frac{1}{\rho^{2}} \frac{c}{4 f^{2}}, \\
f_{3}^{*}=\frac{1}{\rho^{2}}\left(\frac{c}{4 f^{2}}-\left(\frac{f^{\prime}}{f}+\frac{\rho^{\prime}}{\rho}\right)^{2}+\frac{\rho^{\prime} f^{\prime}}{\rho f}+\frac{f^{\prime \prime}}{f}+(\log (\rho))^{\prime \prime}\right) .
\end{gathered}
$$

On the other hand, if we consider on $(M, \phi, \xi, \eta, g)$, a $D$-homothetic deformation defined by

$$
\phi^{*}=\phi, \quad \xi^{*}=\frac{1}{a} \xi, \quad \eta^{*}=a \eta, \quad g^{*}=a g+a(a-1) \eta \otimes \eta,
$$

where $a$ is a positive constant, we can prove:

Theorem 4.3. ([1]) Given a complex-space-form $N(c)$, a positive constant $a$ and a function $f=f(t)>0$, the $D$-homothetic deformation with constant a endows the warped product $M=\mathbb{R} \times_{f} N(c)$ with the structure of a generalized Sasakian-spaceform $M\left(f_{1}^{*}, f_{2}^{*}, f_{3}^{*}\right)$ with functions

$$
f_{1}^{*}=\frac{a c-4 f^{\prime 2}}{4 a^{2} f^{2}}, \quad f_{2}^{*}=\frac{c}{4 a f^{2}}, \quad f_{3}^{*}=\frac{a c-4 f^{\prime 2}}{4 a^{2} f^{2}}+\frac{f^{\prime \prime}}{a^{2} f} .
$$

Finally, if we consider on $M=\mathbb{R} \times{ }_{f} N(c)$ the $D$-conformal deformation given by

$$
\phi^{*}=\phi, \quad \xi^{*}=\frac{1}{\delta^{2}} \xi, \quad \eta^{*}=\delta^{2} \eta, \quad g^{*}=\delta^{2} g+\delta^{2}\left(\delta^{2}-1\right) \eta \otimes \eta,
$$

$\delta=\delta(t)>0$, then we have:

Theorem 4.4. ([1]) Given a complex-space-form $N(c)$ and two functions $f=$ $f(t)>0, \delta=\delta(t)>0$, the D-conformal deformation with function $\delta$ endows the warped product $M=\mathbb{R} \times_{f} N(c)$ with the structure of a generalized Sasakianspace-form $M\left(f_{1}^{*}, f_{2}^{*}, f_{3}^{*}\right)$ with functions

$$
\begin{gathered}
f_{1}^{*}=\frac{1}{\delta^{2}}\left(\frac{c}{4 f^{2}}-\frac{1}{\delta^{2}}\left(\frac{f^{\prime}}{f}+\frac{\delta^{\prime}}{\delta}\right)^{2}\right), \quad f_{2}^{*}=\frac{c}{4 \delta^{2} f^{2}} \\
f_{3}^{*}=\frac{1}{\delta^{2}}\left(\frac{c}{4 f^{2}}-\frac{1}{\delta^{2}}\left(\left(\frac{f^{\prime}}{f}+\frac{\delta^{\prime}}{\delta}\right)^{2}-\left(\frac{f^{\prime \prime}}{f}+\frac{\delta^{\prime \prime}}{\delta}\right)+2\left(\frac{\delta^{\prime}}{\delta}\right)^{2}\right)\right) .
\end{gathered}
$$




\section{Trans-Sasakian generalized Sasakian-space-forms}

First of all, let us point out why trans-Sasakian generalized Sasakian-spaceforms are interesting:

Proposition 5.1. ([1]) Let $N$ be an almost Hermitian manifold. Then, $\mathbb{R} \times_{f} N$ is $a(0, \beta)$ trans-Sasakian manifold, with $\beta=f^{\prime} / f$, if and only if $N$ is a Kaehlerian manifold.

Therefore, the useful warped products $\mathbb{R} \times_{f} N(c)$ of the previous section are examples of trans-Sasakian generalized Sasakian-space-forms.

Actually, as we already said, the important examples of trans-Sasakian manifolds are either $\alpha$-Sasakian or $\beta$-Kenmotsu manifolds.

By working with the second Bianchi identity and after some very long calculations, we can show the following results concerning $\alpha$-Sasakian generalized Sasakianspace-forms:

Proposition 5.2. ([2]) Let $M\left(f_{1}, f_{2}, f_{3}\right)$ be an $\alpha$-Sasakian generalized Sasakianspace-form, with dimension greater than or equal to 5 . Then $\alpha$ depends only on the direction of $\xi$ and the functions $f_{1}, f_{3}$ and $\alpha$ satisfy the equation $f_{1}-f_{3}=\alpha^{2}$.

Theorem 5.1. ([2]) Let $M\left(f_{1}, f_{2}, f_{3}\right)$ be a connected $\alpha$-Sasakian generalized Sasakian-space-form, with dimension greater than or equal to 5 . Then, $f_{1}$ and $f_{2}$ are constant functions and, if either $\alpha=0$ or $\alpha \neq 0$ at every point of $M$, then $f_{3}$ is also a constant function.

With respect to $\beta$-Kenmotsu generalized Sasakian-space-forms, we have:

Proposition 5.3. ([2]) Let $M\left(f_{1}, f_{2}, f_{3}\right)$ be a $\beta$-Kenmotsu generalized Sasakianspace-form. Then, $\beta$ depends only on the direction of $\xi$ and the functions $f_{1}, f_{3}$ and $\beta$ satisfy the equation $f_{1}-f_{3}+\xi(\beta)+\beta^{2}=0$.

Theorem 5.2. ([1]) Let $M\left(f_{1}, f_{2}, f_{3}\right)$ be a $\beta$-Kenmotsu generalized Sasakian-spaceform, with dimension greater than or equal to 5 . Then, $X\left(f_{i}\right)=0$ for any $X$ orthogonal to $\xi, i=1,2,3$, and the following equations hold:

$$
\xi\left(f_{1}\right)+2 \beta f_{3}=0, \quad \xi\left(f_{2}\right)+2 \beta f_{2}=0 .
$$

Actually, by integrating with respect to $t$ in the above equations, we deduce that, locally,

$$
f_{1}=\tilde{F}_{1}-2 \int \beta f_{3} d t, \quad f_{2}=\tilde{F}_{2} e^{-2 \int \beta d t},
$$

where $\partial \tilde{F}_{1} / \partial t=\partial \tilde{F}_{2} / \partial t=0$. 


\section{References}

[1] P. Alegre, D. E. Blair and A. Carriazo, Generalized Sasakian-space-forms, Israel J. Math. 141 (2004), 157-183.

[2] P. Alegre, D. E. Blair and A. Carriazo, Generalized Sasakian-space-forms II, preprint.

[3] D. E. Blair, Riemannian Geometry of Contact and Symplectic Manifolds, Birkhäuser, Boston, 2002.

[4] D. E. Blair, T. Koufogiorgos and B. J. Papantoniou, Contact metric manifolds satisfying a nullity condition, Israel J. Math. 91 (1995), 189-214.

[5] P. Bueken and L. Vanhecke, Curvature characterizations in contact geometry, Riv. Mat. Univ. Parma (4) 14 (1988), 303-313.

[6] J. C. Marrero, The local structure of trans-Sasakian manifolds, Ann. Mat. Pura Appl. 162 (1992), 77-86.

[7] J. A. Oubiña, New classes of almost contact metric structures, Publ. Math. Debrecen 32 (1985), 187-193.

[8] F. Tricerri and L. Vanhecke, Curvature tensors on almost Hermitian manifolds, Trans. Amer. Math. Soc. 267 (1981), 365-398.

[9] L. Vanhecke, Almost Hermitian manifolds with $J$-invariant Riemann curvature tensor, Rend. Sem. Mat. Univ. Politec. Torino 34 (1975-76), 487-498. 